\title{
Cancellative medial groupoids and arithmetic means
}

\section{T. Howroyd}

It is shown that a homomorphism from a commutative, idempotent and medial groupoid with a reducible set of generators into another medial groupoid may be characterized by certain simultaneous equations. This result is used to characterize the arithmetic mean without introducing either continuity or order.

A characterization of the arithmetic mean on the real line was first given by Kolmogoroff [3] and independently by Nagumo [4]. Later Aczél [1] showed that if a compact interval is equipped with a jointly continuous binary operation which is commutative, cancellative, idempotent and medial, then the resulting groupaid is isomorphic and homeomorphic to the closed interval $[0,1]$ under arithmetic mean. Fuchs [2] showed that Aczél's result holds for certain totally ordered groupoids. Sigmon [5] extended Aczél's result to the $n$-dimensional case.

A groupoid is a set together with a binary operation here denoted by multiplication. A groupold is medial if the equation $(x y)(u v)=(x u)(y v)$ is an identity; cancellative if the maps $x+x u$ and $x+u x$ are one-toone for each element $u$; and idempotent if each element is idempotent $(u u=u)$. A subset $A$ of a groupoid is reducible if given $a, b$ and $c$ in $A$ there exists $d$ in $A$ such that $a b=c d$ or $b c=a d$ or $c a=b d$. If $A$ has two elements then $A$ is reducible, if $A$ is a three element subset of a quasigroup $G$ then a fourth element can be added to $A$ to produce a reducible set in $G$.

If $G$ is a groupoid and $A, B$ are subsets of $G$ we write

Received 25 July 1972. 
$A B=\{a b: a \in A$ and $b \in B\}$ and $A^{n+1}=A A^{n},(n=1,2, \ldots)$. We denote the closed interval $[0,1]$ together with the arithmetic mean by $Q$, and the binary fractions in $[0,1]$ with the arithmetic mean by $Q_{0}$. Both $Q$ and $Q_{0}$ are commutative, idempotent, cancellative and medial groupoids.

LEMMA. Let $G$ be a commutative, idempotent and medial groupoid. If $A$ generates $G$ and $A$ is reducible then $G=A G=\bigcup^{\infty} A^{n}$.

Proof. Obviously $A \subset A^{2}$ and by induction $A^{n} \subset A^{n+1}$ for all $n$. We now prove that $A^{n} A^{n}=A^{n+1}$, whence $A^{n} A^{n+m} \subset A^{n+m+1}$ and $\cup A^{n}$ is a subgroupoid of $G$ which contains $A$ and so equals $G$. Now $A \subset A^{n}$ implies $A^{n+1} \subset A^{n} A^{n}$. Trivially $A A \subset A^{2}$. If $A^{n} A^{n} \subset A^{n+1}$ then, since $A$ is reducible,

$$
\begin{aligned}
A^{n+1} A^{n+1} & =\left(A A^{n}\right)\left(A A^{n}\right)=(A A)\left(A^{n} A^{n}\right) \\
& \subset(A A)\left(A A^{n}\right) \subset A\left(A A^{n}\right)=A^{n+2},
\end{aligned}
$$

and the result follows by induction. Finally

$$
A G=A \bigcup_{2}^{\infty} A^{n}=\bigcup_{1}^{\infty} A^{n}=G .
$$

THEOREM 1. Let $G$ be a commutative, idempotent and medial groupoid. Let $A$ generate $G$ and be reducible. Let $B$ be a medial groupoid. Then $\phi: G \rightarrow H$ is a homomorphism if and only if

$$
\phi(\alpha x)=\phi(\alpha) \phi(x) \text { for all } \alpha \text { in } A \text { and } x \text { in } G \text {. }
$$

Proof. The "only if" part is trivial. Let us suppose (I) is true. We first prove that

$$
\phi(\xi n)=\phi(\xi) \phi(n) \text { for all } \xi \text { and } \eta \text { in } A^{2} \text {. }
$$

Let $\xi=\alpha \beta$ and $\eta=\gamma \delta$ where $\alpha, \beta, \gamma$ and $\delta$ are in $A$. Then

$$
\phi((\alpha \beta)(\gamma \delta))=\phi((\alpha \gamma)(\beta \delta))=\phi((\beta \gamma)(\alpha \delta))
$$


and

$$
\begin{aligned}
\phi(\alpha \beta) \phi(\gamma \delta) & =(\phi(\alpha) \phi(\beta))(\phi(\gamma) \phi(\delta)) \\
& =(\phi(\alpha) \phi(\gamma))(\phi(\beta) \phi(\delta)) \\
& =\phi(\alpha \gamma) \phi(\beta \delta)=\phi(\beta \gamma) \phi(\alpha \delta) .
\end{aligned}
$$

Hence when using the reducibility of $A$ we may assume there exists $\nu$ in $A$ such that $\alpha \beta=\gamma \nu$, so

$$
\begin{aligned}
\phi((\alpha \beta)(\gamma \delta)) & =\phi(\gamma(\nu \delta))=\phi(\gamma)(\phi(\nu) \phi(\delta)) \\
& =(\phi(\gamma) \phi(\gamma))(\phi(\nu) \phi(\delta)) \\
& =(\phi(\gamma) \phi(\nu))(\phi(\gamma) \phi(\delta)) \\
& =\phi(\gamma \nu) \phi(\gamma \delta) \\
& =\phi(\alpha \beta) \phi(\gamma \delta) .
\end{aligned}
$$

We now prove that if $\xi \in A^{2}$ and $x \in G$ then

$$
\phi(\xi x)=\phi(\xi) \phi(x) \text {. }
$$

If $\delta \in A, \xi, \eta \in A^{2}$, and $x \in G$ then

$$
\phi(n \delta)(\phi(\xi) \phi(x))=\phi(\xi n) \phi(\delta x)
$$

since

$$
\begin{aligned}
\text { LHS } & =(\phi(\eta) \phi(\delta))(\phi(\xi) \phi(x)) \\
& =(\phi(\eta) \phi(\xi))(\phi(\delta) \phi(x))=\text { RHS } .
\end{aligned}
$$

Let $\xi \in A^{2}$ and $x \in G$. Then there exist $\alpha$ in $A$ and $\theta$ in $G$ such that $x=\alpha s$; and since $A$ is reducible there exist $\delta$ and $\mu$ in $A$ such that $\xi(\alpha y)=\delta(\mu y)$ for all $y$ in $G$. Hence writing $\eta=\alpha \mu$ we have $\xi \alpha=\delta n$ and $\delta \mu=\xi \eta$,

$$
\begin{aligned}
\phi(\xi x) & =\phi(\delta(\mu s))=\phi(\delta)(\phi(\mu) \phi(s)) \\
& =(\phi(\delta) \phi(\mu))(\phi(\delta) \phi(s)) \\
& =\phi(\delta \mu) \phi(\delta s)=\phi(\xi n) \phi(\delta s) \\
& =\phi(n \delta)(\phi(\xi) \phi(s)) \quad(\text { by }(3)) \\
& =\phi(\xi \alpha)(\phi(\xi) \phi(s)) \\
& =\phi(\xi) \phi(\alpha s) \\
& =\phi(\xi) \phi(x) .
\end{aligned}
$$

Let $n$ be a positive integer such that $\phi(x y)=\phi(x) \phi(y)$ for all $x$ and $y$ in $A^{n}$. If $\alpha, \beta \in A$ and $s, t \in A^{n}$ then 


$$
\begin{aligned}
\phi((\alpha \beta)(\beta t)) & =\phi((\alpha \beta)(s t))=\phi(\alpha \beta) \phi(s t) \\
& =(\phi(\alpha) \phi(\beta))(\phi(s) \phi(t)) \\
& =(\phi(\alpha) \phi(s))(\phi(\beta) \phi(t)) \\
& =\phi(\alpha s) \phi(\beta t) .
\end{aligned}
$$

It follows by induction that $\phi$ is a homomorphism.

THEOREM 2. Let $G$ be a commutative, cancellative, idempotent and medial groupoid generated by the elements $a$ and $b$ so that $a x=$ by only if $x=b$ and $y=a$. Then $G$ is isomorphic to $Q_{0}$.

Proof. Let $A=\{a, b\}$. We define $\phi: G \rightarrow Q_{0}$ so that $\phi(a)=0$, $\phi(b)=I$, and inductively extend the domain of $\phi$ to $G$ so that the equations

$$
\begin{aligned}
& \phi(a x)=\frac{1}{2} \phi(x), \\
& \phi(b x)=\frac{1}{2}+\frac{1}{2} \phi(x)
\end{aligned}
$$

are valid for all $x \in A^{n}$ and each $n$. Let $S=\{0,1\}$ and $n S=\left\{\frac{1}{2} x+\frac{1}{2} y: x \in S, y \in(n-1) S\right\},(n=2,3, \ldots)$. Then (4) and (5) obviously well define $\phi$ as a one-to-one map of $A^{2}$ onto $2 S$.

Suppose (4) and (5) well define $\phi$ as a one-to-one map of $A^{p}$ onto $p S$. If $x$ is in $A^{p} \backslash A^{p-1}$ then $a x$ and $b x$ are in $A^{p+1} \backslash A^{p}, \phi(x)$ is in $p S \backslash(p-1) S$ and the numbers $\frac{k}{2} \phi(x)$ and $\frac{1}{2}+\frac{3}{2} \phi(x)$ are distinct elements of $(p+1) S \backslash p S$. Hence (4) and (5) well define $\phi$ as a one-to-one map of $A^{p+1}$ onto $(p+1) S$. But $G=U A^{n}$ and $Q_{0}=U(n S)$. Hence $\phi$ is a well defined one-to-one map of $G$ onto $Q_{0}$ and $\phi$ satisfies (4) and (5). These equations correspond to (1) in Theorem 1 . Hence $\phi$ is an isomorphism.

Note 1. Let $G$ be the compect interval $[a, b]$ together with a jointly continuous binary operation which is commutative, cancellative, idempotent and medial. Then $a G$ and $b G$ have only the element $a b$ in common. The binary operation is intern $(x<x y<y)$, so $U\{a, b\}^{n}$ is dense in $G$. The isomorphism constructed in Theorem 2 may be shown to be strictly increasing and extended to ell of $G$ whence $G$ is iscmorphic to $Q$ as in [1]. 
Note 2. Soublin ([6], p. 106) has shown that a cancellative and distributive $(x(u v)=(x u)(x v))$ groupoid with two or three generators is medial. It follows that Theorem 2 remains true if "medial" is replaced by "distributive" (cf. [6], p. 253); then of course it is well known that a cancellative and distributive groupoid is idempotent so that "idempotent" may be removed from Theorem 2 .

\section{References}

[1] J. Aczél, "On mean values", Bull. Amer. Math. Soc. 54 (1948), 392-400.

[2] L. Fuchs, "On mean systems", Acta Math. Acad. Sci. Hungar. 1 (1950), 303-320.

[3] A. Kolmogoroff, "Sur la notion de la moyenne", Atti. Acad. Naz. Lincei Rend. (6) 12 (1930), 388-391.

[4] M. Nagumo, "Über eine Klasse der Mittelwerte", Japan J. Math. 7 (1930), 71-79.

[5] Kermit Sigmon, "Cancellative medial means are arithmetic", Duke Math. J. 37 (1970), 439-445.

[6] Jean-Pierre Soublin, "Étude algébrique de la notion de moyenne", $J$. Math. Pures App 2. (9) 50 (1971), 53-264.

Department of Mathematics, University of New Brunswick, Fredericton, New Brunswick, Canada. 